\title{
Calculating Radiation Temperature Anisotropy in Flat Space Cosmology
}

\author{
Eugene Terry Tatum \\ 760 Campbell Ln. Ste. 106 \#161, Bowling Green, KY, USA \\ Email:ett@twc.com
}

How to cite this paper: Tatum, E.T. (2018) Calculating Radiation Temperature Anisotropy in Flat Space Cosmology. Journal of Modern Physics, 9, 1946-1953. https://doi.org/10.4236/jmp.2018.910123

Received: July 12, 2018

Accepted: September 1, 2018

Published: September 4, 2018

Copyright $\odot 2018$ by author and Scientific Research Publishing Inc. This work is licensed under the Creative Commons Attribution International License (CC BY 4.0).

http://creativecommons.org/licenses/by/4.0/

\section{(c) (i) Open Access}

\begin{abstract}
The purpose of this paper is to show how one can use the FSC model of gravitational entropy to calculate cosmic radiation temperature anisotropy for any past cosmic time $t$ since the Planck scale. Cosmic entropy follows the Bekenstein-Hawking definition, although in the correct-scaling form of $\sqrt{S}$, which scales 60.63 logs of 10 from the Planck scale. In the FSC model, cosmic radiation temperature anisotropy $A_{t}=\left(t / t_{o}\right)$. The derived past anisotropy value can be compared to current co-moving anisotropy defined as unity $\left(t_{o} / t_{o}\right)$. Calculated in this way, current gravitational entropy and temperature anisotropy have maximum values, and the earliest universe has the lowest entropy and temperature anisotropy values. This approach comports with the second law of thermodynamics and the theoretical basis of the Sachs-Wolfe effect, gravitational entropy as defined by Roger Penrose, and Erik Verlinde's "emergent gravity" theory.
\end{abstract}

\section{Keywords}

Flat Space Cosmology, Cosmic Microwave Background, CMB Anisotropy, Cosmology Theory, Cosmic Entropy, Gravitational Entropy, Black Holes, Standard Cosmology

\section{Introduction and Background}

In the July 2018 issue of Journal of Modern Physics, the paper entitled, "How the CMB Anisotropy Pattern Could Be a Map of Gravitational Entropy" [1] presents the rationale for Flat Space Cosmology (FSC) calculations of gravitational entropy in the form of $\sqrt{S}$. The theoretical basis for doing so is the Sachs-Wolfe effect [2]. The Sachs-Wolfe effect is widely considered to be the source of large angular scale temperature fluctuations in the cosmic microwave background (CMB). However, in a spatially flat universe, the Sachs-Wolfe effect can also be 
considered to be the source of the smaller angular scale fluctuations of the CMB temperature anisotropy [3]. The Boomerang Collaboration [4] reported CMB anisotropy observations closely fitting "the theoretical predictions for a spatially flat cosmological model with an exactly scale invariant primordial power spectrum for the adiabatic growing mode" [Bucher (2015), page 6]. Thus, this theoretical basis for the Sachs-Wolfe effect as a measure of "gravitational potential" appears to explain the tight correlation between the FSC CMB anisotropy calculations and the observed CMB anisotropy [5] [6] [7].

Furthermore, the FSC CMB anisotropy paper and its companion paper [8] in the July 2018 Journal of Modern Physics show how the FSC model dovetails nicely with Erik Verlinde's concept of "emergent gravity" as an emergent property of cosmic entropy [9] [10] and Roger Penrose's concept of gravitational entropy [11] based upon his "Weyl's curvature hypothesis" [12] [13].

The purpose of this paper is to show how the FSC CMB anisotropy paper opens the way for a definition of cosmic radiation temperature anisotropy at any cosmic temperature $T$, whether it is in the form of "Universal Temperature" $T_{U}$ s as defined in FSC reference [14], or in the Kelvin temperature scale. Although the FSC CMB anisotropy paper shows how to calculate the gravitational entropy ratios relating entropies at given years of cosmic time since the Planck epoch, these values can also be calculated in given seconds of cosmic time since the Planck epoch. This allows for the first year cosmic times and gravitational entropies to be correlated second-by-second as shown below (see Equation (3)). Therefore, cosmic radiation temperature anisotropy is not only predicted by FSC for the $\mathrm{CMB}$ recombination/decoupling "last scattering surface" but also for any other cosmic time $t$.

The rationale for generalizing the radiation temperature anisotropy calculations to be presented herein can be summarized as follows: Sachs and Wolfe used a gravitational redshift theoretical argument that radiation temperature anisotropy could be a result of inhomogeneous gravitational particle clustering. So, while they applied their argument in anticipation of refined CMB anisotropy observations, there was nothing particularly special about the CMB emission event with respect to their gravitational redshift argument. The recombination/decoupling event concerned photon emission, making the event observable. However, the coupling of electrons with protons to form the first hydrogen atoms should have no impact whatsoever on gravitational particle clustering. As explained in reference [1], the Sachs-Wolfe effect is now widely considered to be the theoretical basis for large angular scale radiation temperature fluctuations. Furthermore, in a spatially flat universe, the Sachs-Wolfe effect can also be considered to be the source of the smaller angular scale fluctuations of the CMB temperature anisotropy [3]. Therefore, based upon this scale-invariant flat universe Sachs-Wolfe effect, the calculation method presented in reference [1] and herein is believed to be generalizable to any other gravitational particle clustering stage (i.e., gravitational entropy stage) of universal expansion. 
The reader will maximally benefit in reading the present paper after first reading FSC references [1] [8] and [14]. FSC is a mathematical model of the Hawking-Penrose conjecture that a universe smoothly expanding from a singularity can be treated, under general relativity, much like a time-reversal of giant black hole collapse to a singularity. A brief introductory review of the FSC assumptions, and their justification, is provided below. The five assumptions of FSC are:

\section{The Five Assumptions of Flat Space Cosmology}

1) The cosmic model is an ever-expanding sphere such that the cosmic horizon always translates at speed of light $c$ with respect to its geometric center at all times $t$. The observer is operationally-defined to be at this geometric center at all times $t$.

2) The cosmic radius $R_{t}$ and total mass $M_{t}$ follow the Schwarzschild formula $R_{t} \cong 2 G M_{t} / c^{2}$ at all times $t$.

3) The cosmic Hubble parameter is defined by $H_{t} \cong c / R_{t}$ at all times $t$.

4) Incorporating our cosmological scaling adaptation of Hawking's black hole temperature formula, at any radius $R_{t}$ cosmic temperature $T_{t}$ is inversely proportional to the geometric mean of cosmic total mass $M_{t}$ and the Planck mass $M_{p l} . R_{p l}$ is defined as twice the Planck length (i.e., as the Schwarzschild radius of the Planck mass black hole). With subscript $t$ for any time stage of cosmic evolution and subscript $p l$ for the Planck scale epoch, and, incorporating the Schwarzschild relationship between $M_{t}$ and $R_{t}$,

$$
\begin{aligned}
& \left.k_{B} T_{t} \cong \frac{\hbar c^{3}}{8 \pi G \sqrt{M_{t} M_{p l}}} \cong \frac{\hbar c}{4 \pi \sqrt{R_{t} R_{p l}}}\right) \\
& \left\{\begin{array}{ll}
M_{t} \cong\left(\frac{\hbar c^{3}}{8 \pi G k_{B} T_{t}}\right)^{2} \frac{1}{M_{p l}} & \text { (A) } \\
R_{t} \cong \frac{1}{R_{p l}}\left(\frac{\hbar c}{4 \pi k_{B}}\right)^{2}\left(\frac{1}{T_{t}}\right)^{2} & \text { (B) } \\
R_{t} T_{t}^{2} \cong \frac{1}{R_{p l}}\left(\frac{\hbar c}{4 \pi k_{B}}\right)^{2} & \text { (C) } \\
t \cong \frac{R_{t}}{c} & \text { (D) }
\end{array}\right\}
\end{aligned}
$$

5) Total entropy of the cosmic model follows the Bekenstein-Hawking black hole formula [13] [14].

$$
S_{t} \cong \frac{\pi R_{t}^{2}}{L_{p}^{2}}
$$

The first two assumptions are based upon a literal interpretation of the Hawking-Penrose conjecture as it would pertain to a smoothly-expanding Schwarzschild black hole. The third assumption (Hubble parameter) treats maximally redshifted radial photons at the cosmic model horizon as moving 
with speed of light $c$ relative to the geometric center at a distance of horizon radius $R_{t}$. This is a stipulation of relativity. The fourth assumption is a cosmic temperature scaling assumption. While it shows similarity to the static Hawking black hole temperature formula, the FSC cosmic model is treated as scaling in Planck mass increments. This allows for dynamic cosmic expansion modeling from the Planck scale epoch. Finally, the fifth assumption utilizes the Bekenstein-Hawking entropy definition, which seems appropriate for a model of the Hawking-Penrose conjecture. The numerous observational correlations of FSC are given in references [8] and [14].

\section{Calculating Radiation Temperature Anisotropy in FSC}

Equation (11) from reference [14] gives an FSC cosmic time value of $10^{11.58}$ seconds at the beginning of the recombination/decoupling epoch $(3000 \mathrm{~K})$. This equation is repeated here as Equation (3):

$$
T^{2} t_{s}=3.426525959553982 \times 10^{18} \mathrm{~K}^{2} \cdot \mathrm{s}
$$

wherein cosmic temperature $T$ is in degrees $K$ and cosmic time $t_{s}$ is in seconds since the Planck epoch. For reasons given in the reference [1] Discussion section, the "end of decoupling" event happened in the FSC model at $10^{12.6}$ seconds (at a temperature of $924.63 \mathrm{~K}$ ) after the Planck epoch. The Planck epoch is the cosmic time of the Planck-scale universe and is often considered to be the approximate moment of the "Big Bang" in standard cosmology. FSC reference [8] derives

$$
\sqrt{S}=\frac{c \sqrt{\pi}}{L_{p}} t
$$

showing the direct proportionality relationship between gravitational entropy $\sqrt{S}$ and cosmic time $t$. Speed of light $c$ and Planck length $L_{p}$ are assumed to be constants over cosmic time. Thus, if we normalize the proportionality constant to unity and operationally define $\sqrt{S}$ in terms of seconds,

$\sqrt{S}=10^{-42.965}$ at $10^{-42.965}$ second of cosmic time at Planck temperature $5.6 \times$ $10^{30} \mathrm{~K}$

$\sqrt{S}=10^{11.58}$ at $10^{11.58}$ seconds of cosmic time at CMB beginning temperature $3000 \mathrm{~K}$;

$\sqrt{S}=10^{12.6}$ at $10^{12.6}$ seconds of cosmic time at end-of-decoupling temperature $924.63 \mathrm{~K}$;

$\sqrt{S}=10^{17.66}$ at $10^{17.66}$ seconds of cosmic time at current temperature 2.72548 K.

The above $\mathrm{CMB}$ emission epoch gravitational entropies $\left(10^{11.58}\right.$ and $\left.10^{12.6}\right)$ can then be related to current cosmic entropy $\left(10^{17.66}\right)$, in ratio form, as follows:

$[\sqrt{S}$ at the beginning of $\mathrm{CMB}$ emission $] /[\sqrt{S}$ at current time $]=8.25 \times$ $10^{-7}\left(0.825 \times 10^{-6}\right)$;

[ $\sqrt{S}$ at the ending of $\mathrm{CMB}$ emission] $/[\sqrt{S}$ at current time $]=8.69 \times 10^{-6}$ $\left(0.869 \times 10^{-5}\right)$. 
These derived values of the beginning and ending CMB radiation temperature anisotropy are the same as those calculated in reference [1]. This value range fits the COBE DMR CMB $d T / T$ anisotropy measurement of $0.66 \times 10^{-5}$, as well as with the WMAP and Planck report anisotropy estimates of "approximately" $10^{-5}$.

Thus, if this process for calculating the $\mathrm{CMB}$ radiation temperature anisotropy as gravitational entropy ratios can be generalized and extended all the way back to the Planck epoch (and conceivably beyond), the formula for doing such calculations is:

$$
A_{t}=\sqrt{S}_{t} / \sqrt{S}_{o}=t / t_{o}
$$

wherein $A_{t}$ is the radiation temperature anisotropy at cosmic time $t, \sqrt{S}_{t}$ is the gravitational entropy (cosmic entropy) at cosmic time $t, \sqrt{S}_{o}$ is the gravitational entropy (cosmic entropy) at current time $t_{o}$, and $t / t_{o}$ is the ratio of these time values. This radiation temperature anisotropy formula can also be substituted by other FSC parameters correlated to $\sqrt{S}_{t}$, as seen in reference [8]. Thus,

$$
A_{t}=R_{t} / R_{o}
$$

wherein $R_{t}$ is the cosmic radius at cosmic time $t$ and $R_{o}$ is the current observed cosmic radius.

$$
A_{t}=T_{o}^{2} / T_{t}^{2}
$$

wherein $T_{o}$ is the current cosmic radiation temperature in degrees Kelvin $(2.72548 \mathrm{~K})$ and $T_{t}$ is the cosmic radiation temperature in degrees Kelvin at cosmic time $t$.

$$
A_{t}=T_{U o} / T_{U t}
$$

wherein $T_{U o}$ is the current cosmic radiation temperature in Universal Temperature units and $T_{U t}$ is the cosmic radiation temperature in Universal Temperature units at cosmic time $t$. As indicated in reference [14], $T_{U t}$ is defined by $T_{U t}=T_{t}^{2}$.

Based upon this calculation method, the following Figure 1 and Figure 2 can be presented. For comparison, the reader is referred to Figure 1 and Figure 5 in reference [14]. The lines are linear because the vertical and horizontal axes scale in a logarithmic fashion.

Figure 1 shows how radiation temperature anisotropy and total cosmic entropy in the form of $\sqrt{S}$ scales with respect to cosmic time $t$ and Kelvin temperature $T$. CMB starting and ending values are denoted by the white circles.

Figure 2 shows how radiation temperature anisotropy and total cosmic entropy in the form of $\sqrt{S}$ scales with respect to cosmic time $t$ and Universal Temperature $T_{U}$. CMB starting and ending values are denoted by the white circles.

\section{Discussion}

Extensive comparisons between FSC and standard inflationary cosmology are given in reference [15]. Standard inflationary cosmology has no theoretical basis 


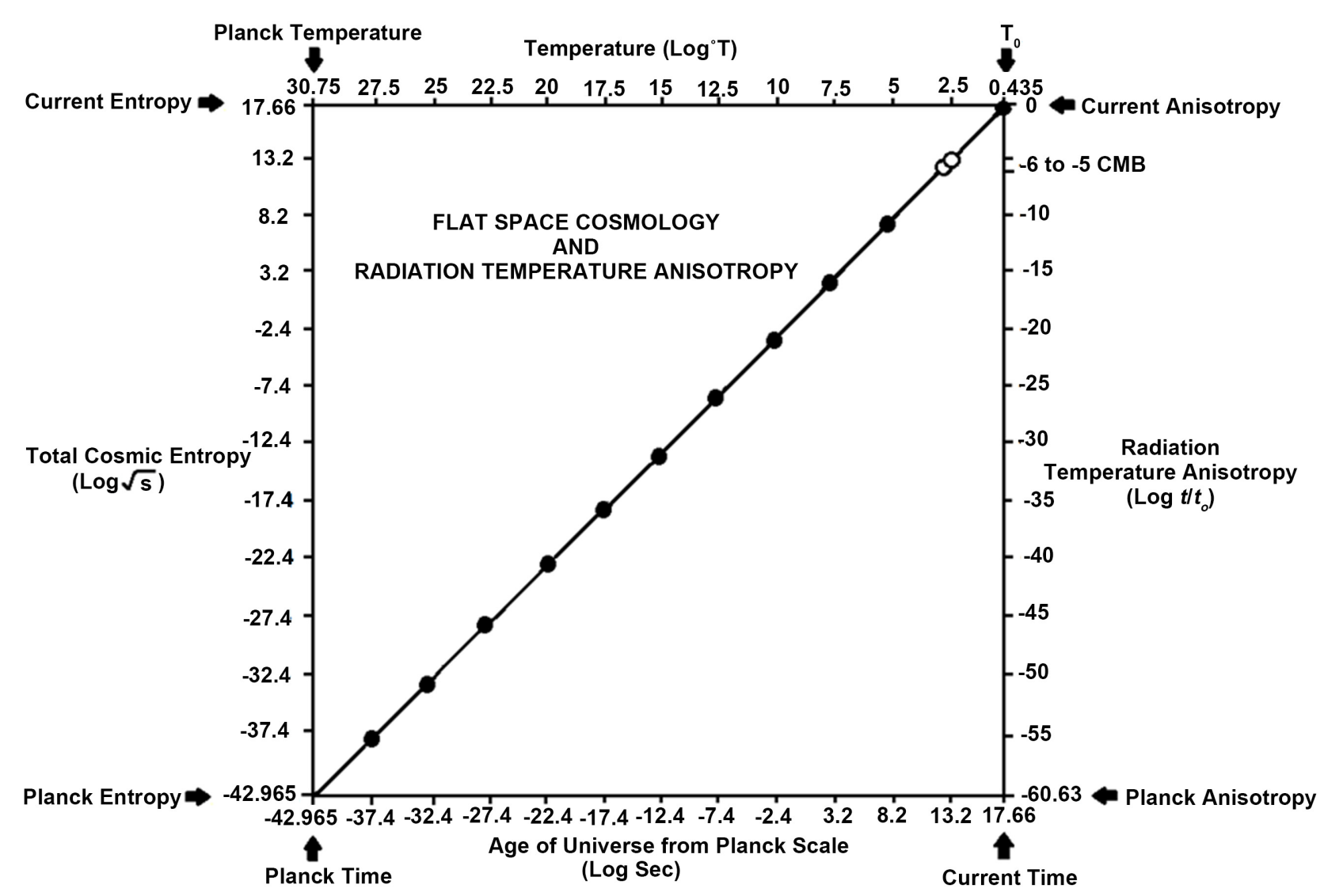

Figure 1. Total entropy, Kelvin temperature T, and temp anisotropy.

for predicting and calculating radiation temperature anisotropy at any given cosmic time. Thus, standard model practitioners can only guess as to the possible reasons why the CMB anisotropy pattern has a $d T / T$ RMS anisotropy value of 18 micro-Kelvins $/ 2.725 \mathrm{~K}=0.66 \times 10^{-5}$, as measured in the COBE DMR experiment [Wright (1996)]. Current speculation seems to favor a CMB pattern produced by a "quantum fluctuation" Big Bang event smoothed out by cosmic inflation and splayed out across the sky. It is even proposed that a "quantum fluctuation" CMB pattern somehow must provide important clues to the nature of gravity at the quantum scale (i.e., "quantum gravity").

In contrast, the FSC model, as detailed in references [1] and [8], indicates that the CMB pattern could simply be a map of gravitational entropy. The theoretical basis for this interpretation owes much to the prior work of Sachs and Wolfe, Hawking, Penrose and Verlinde, as discussed in both FSC papers. Verlinde's papers [9] [10], in particular, address the deep correlation between cosmic entropy and gravity. Thus, given the FSC success in correlating gravitational entropy in the form of $\sqrt{S}$ with the observed anisotropy of the CMB pattern, the current paper proposes a reasonable extension of the same rationale to calculating radiation temperature anisotropy at any past cosmic time $t$ relative to current cosmic anisotropy. For comparison purposes, current co-moving anisotropy can be defined as unity [i.e., $\left.\log \left(t_{o} / t_{o}\right)=0\right]$. 


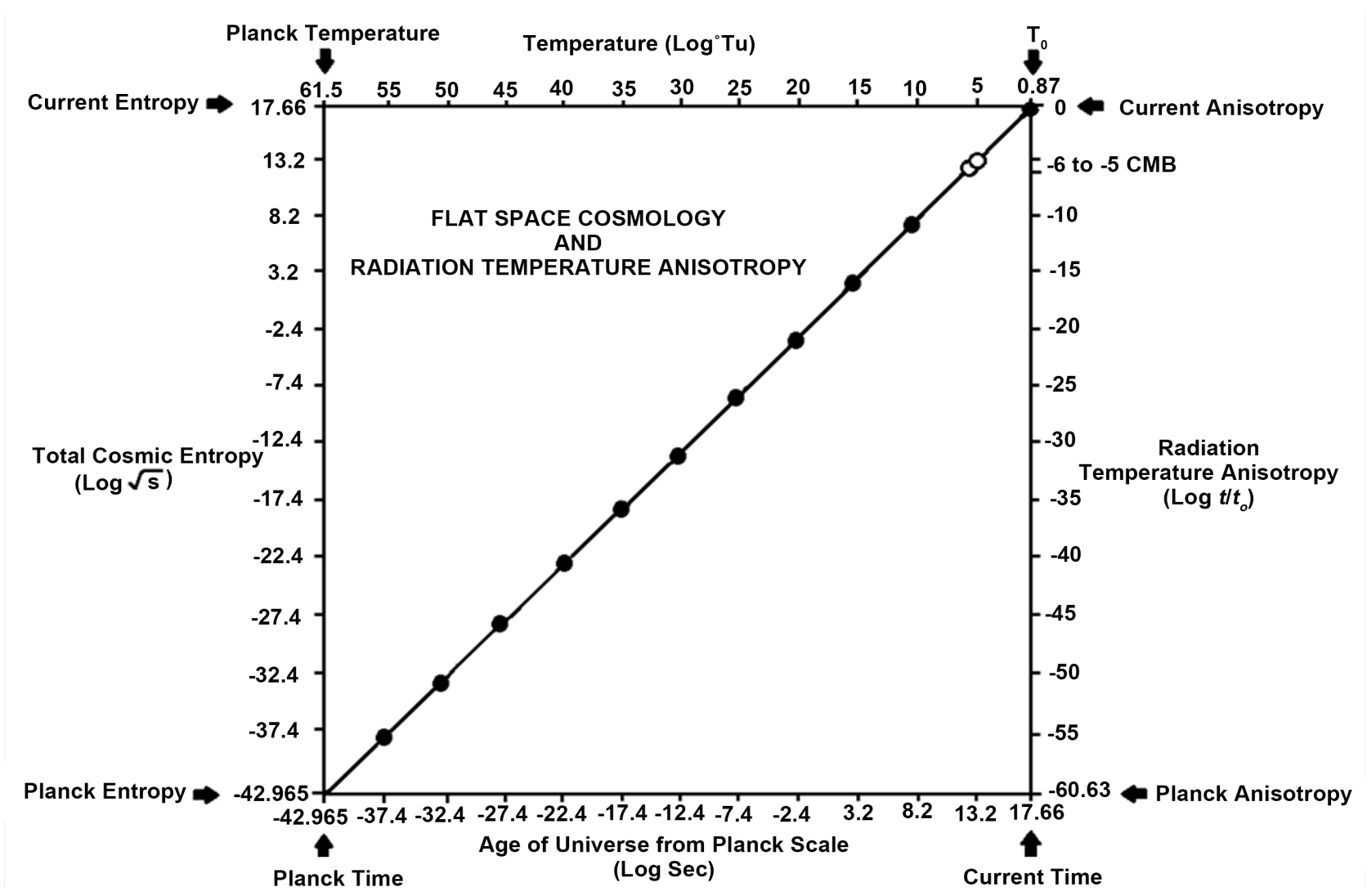

Figure 2. Total entropy, universal temperature $T_{U}$ and temp anisotropy.

Note that, by this definition of current co-moving anisotropy, current gravitational entropy and temperature anisotropy have maximum values, and the earliest universe has the lowest entropy and temperature anisotropy values. This approach comports with the second law of thermodynamics and the theoretical basis of the Sachs-Wolfe effect, gravitational entropy as defined by Roger Penrose, and Erik Verlinde's “emergent gravity" theory.

\section{Summary and Conclusions}

The purpose of this paper has been to show how one can use the FSC model of gravitational entropy to calculate cosmic radiation temperature anisotropy for any past cosmic time $t$ since the Planck scale. In the FSC model, cosmic radiation temperature anisotropy $A_{t}=\left(t / t_{o}\right)$. The derived past anisotropy value can be compared to current co-moving anisotropy defined as unity $\left(\mathrm{t}_{d} / t_{o}\right)$. Calculated in this way, current gravitational entropy and temperature anisotropy have maximum values, and the earliest universe has the lowest entropy and temperature anisotropy values. This approach comports with the second law of thermodynamics and the theoretical basis of the Sachs-Wolfe effect, gravitational entropy as defined by Roger Penrose, and Erik Verlinde's "emergent gravity" theory.

\section{Dedications and Acknowledgements}

This paper is dedicated to the late Dr. Stephen Hawking and Dr. Roger Penrose 
for their groundbreaking work on black holes and their possible application to cosmology. Dr. Tatum also thanks Dr. Rudolph Schild of the Harvard Center for Astrophysics for his past support and encouragement.

\section{Conflicts of Interest}

The author declares no conflicts of interest regarding the publication of this paper.

\section{References}

[1] Tatum, E.T. (2018) Journal of Modern Physics, 9, 1484-1490. https://doi.org/10.4236/jmp.2018.98092

[2] Sachs, R.K. and Wolfe, A.M. (1967) Astrophysical Journal, 147, 73. https://doi.org/10.1086/148982

[3] Bucher, M. (2015) Physics of the Cosmic Microwave Background Anisotropy. arXiv:1501.04288v1 [astro-ph.CO].

[4] De Bernardis, P., et al. (2000) A Flat Universe from High-Resolution Maps of the Cosmic Microwave Background Radiation. arXiv:astro-ph/0004404v1.

[5] Wright, E.L., et al. (1996) Astrophysical Journal, 464, L21-L24. https://doi.org/10.1086/310073

[6] Bennett, C.L. (2013) Nine-Year Wilkinson Microwave Anisotropy Probe (WMAP) Observations: Final Maps and Results. arXiv:1212.5225v3 [astro-ph.CO].

[7] Planck Collaboration. (2014) Astronomy \& Astrophysics, A23, 1-48.

[8] Tatum, E.T. and Seshavatharam, U.V.S. (2018) Journal of Modern Physics, 9, 1469-1483. https://doi.org/10.4236/jmp.2018.98091

[9] Verlinde, E. (2010) On the Origin of Gravity and the Laws of Newton. arXiv:1001.0785v1 [hep-th].

[10] Verlinde, E. (2016) Emergent Gravity and the Dark Universe. aeXiv:1611.02269v2 [hep-th].

[11] Penrose, R. (2016) Fashion Faith and Fantasy in the New Physics of the Universe. Princeton University Press, Princeton, US. https://doi.org/10.1515/9781400880287

[12] Penrose, R. (1979) Singularities and Time-Asymmetry. In: Hawking, S.W. and Israel, W., Eds., General Relativity. An Einstein Centenary Survey, Cambridge University Press, Cambridge, 581-638.

[13] Tatum, E.T. (2018) Journal of Modern Physics, 9, 1935-1940. https://doi.org/10.4236/jmp.2018.910121

[14] Tatum, E.T. and Seshavatharam, U.V.S. (2018) Journal of Modern Physics, 9, 1404-1414. https://doi.org/10.4236/jmp.2018.97085

[15] Tatum, E.T. (2018) Journal of Modern Physics, 9, 1867-1882. https://doi.org/10.4236/jmp.2018.910118 